\title{
A STEM-EELS Study of the Effect of Solar-wind Irradiation on the Ilmenite from Lunar Soil
}

\author{
S. Zhang ${ }^{*}$ and L. P. Keller** \\ *Lunar and Planetary Institute, 3600 Bay Area Blvd., Houston, TX 77058 \\ ***ARES, Mail Code KR, NASA Johnson Space Center, Houston, TX 77058
}

Introduction: The surfaces of fine ilmenite grains from lunar soil are exposed to solarwind irradiation that results in a damaged layer $(\sim 50 \mathrm{~nm})$ showing Fe-Ti segregation [1]. The mechanism responsible for the Fe/Ti distribution in these layers is still unclear. The depth dependent profile of $\mathrm{Fe} / \mathrm{Ti}$ valence variation as well as oxygen distribution within the ilmenite rims would provide critical data to answer this question. In this study, we employed coupled STEM/EELS methods to investigate the correlation between Fe and $\mathrm{Ti}$ valence change with depth in the ilmenite rims. In addition, the effect of solar-wind irradiation on the oxygen variation in these rims was determined.

Materials and experimental procedures: Aliquots of the $<20 \mathrm{~mm}$ fraction of soil 10084 , which was returned by Apollo 11, were embedded in low viscosity epoxy and thin sections ( $\sim 60 \mathrm{~nm}$ thick) were prepared using ultramicrotomy. The STEM-EELS analysis was obtained using the JEOL 2500SE $200 \mathrm{kV}$ field-emission STEM equipped with a Tridiem GIF operated with a $4 \mathrm{~nm}$ probe, $4 \mathrm{~s}$ dwell time, $0.2 \mathrm{eV} /$ channel dispersion, $0.8 \mathrm{eV}$ energy resolution, and $4 \times 4 \mathrm{~nm}$ pixel size.

Results and discussion: In the solar wind irradiated layer of ilmenite, nanophase $(<10 \mathrm{~nm})$ iron metal $\left(\mathrm{npFe}^{0}\right)$ grains occur adjacent to the Ti-rich phase (Fig.1b). Qualitative comparison of Fe/Ti valence with depth across the ilmenite rims can be obtained by calculation of integrated intensity ratio of $\mathrm{L}_{3} / \mathrm{L}_{2}$ for $\mathrm{Fe}$ and Ti, respectively. STEM-EELS mapping of the rim shows the amount of $\mathrm{Ti}^{3+}$ increases towards the surface, and more $\mathrm{Fe}^{2+}$ in ilmenite was reduced to $n \mathrm{pFe}^{0}$ [Fig.2]. This observation is consistent with the preferential loss of oxygen, compared with the Fe/Ti cations in ilmenite, due to solar wind irradiation, as also demonstrated by the depth dependent profile of O/Ti ratio (Fig.2). Thus, the Fe-Ti segregation in the rims of lunar ilmenite likely results from in situ reduction induced by the solar wind irradiation. In this process, with the loss of oxygen, part of $\mathrm{Ti}^{4+}$ and $\mathrm{Fe}^{2+}$ is reduced to $\mathrm{Ti}^{3+}$ and $\mathrm{Fe}$ metal, respectively. 
The stoichiometry of the Ti-enriched phase, i.e. $\mathrm{Ti}^{3+}$-bearing ilmenite or pure $\mathrm{Ti}$ oxide, in the ilmenite rims is intriguing. With negligible over- or undersampling effect as described in the experimental method section, STEM-EELS analysis of the $n p F e^{0}$ commonly shows considerable presence of $\mathrm{Fe}^{2+}$, and a mixture of $\mathrm{Ti}^{3+}$ and $\mathrm{Ti}^{4+}$, which is likely from the Tibearing phase buried underneath the Fe metal. Similarly, the Ti-enriched region was observed to have mixed valence of $\mathrm{Fe}^{2+}$ and $\mathrm{Fe}^{0}$, though occasionally it is simply pure reduced Ti oxide. High resolution TEM examination of the Ti-rich zone typically shows defect microstructures, such as stacking faults, which likely arise from the reconstruction of $\mathrm{Ti} / \mathrm{Fe}$ layer in ilmenite. The seemingly pure $\mathrm{Ti}$ oxide regions are amorphous or nanocrystalline. Therefore, given the fluence of the solar-wind irradiation the lunar ilmenite had experienced, the majority of the in situ reduction products are confined to the $\mathrm{Ti}^{3+}$ bearing intermediate ilmenite and $\mathrm{npFe}^{0}$. Continued bombardment of the rims by the solarwind ions could eventually lead to reduced $\mathrm{Ti}$ oxide in replace of intermediate ilmenite. The mineralogical and chemical analysis of the ilmenite rims in lunar soil, therefore, could provide quantitative constraints on their formation conditions.

Reference: [1] Christoffersen, R. et al. (1996) Meteoritics \& Planet. Sci. 31, 835-848.

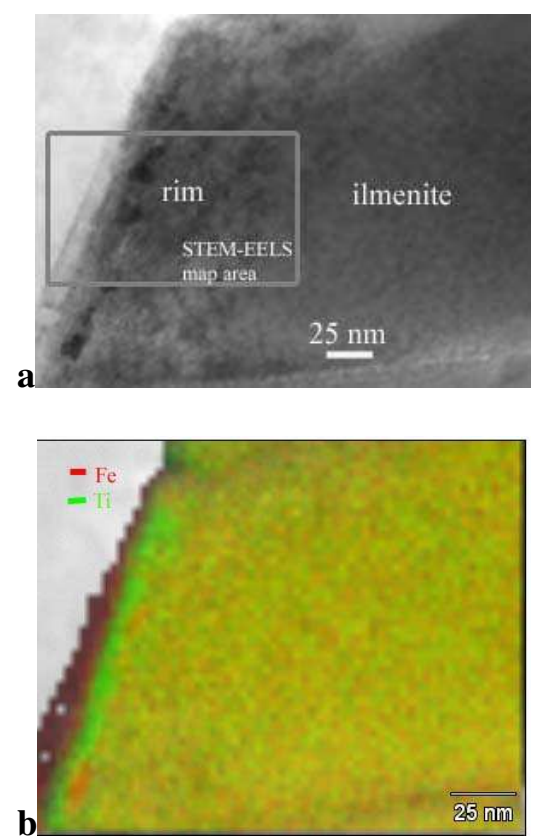

Figure 1. STEM-EDS mapping of the ilmenite rim.

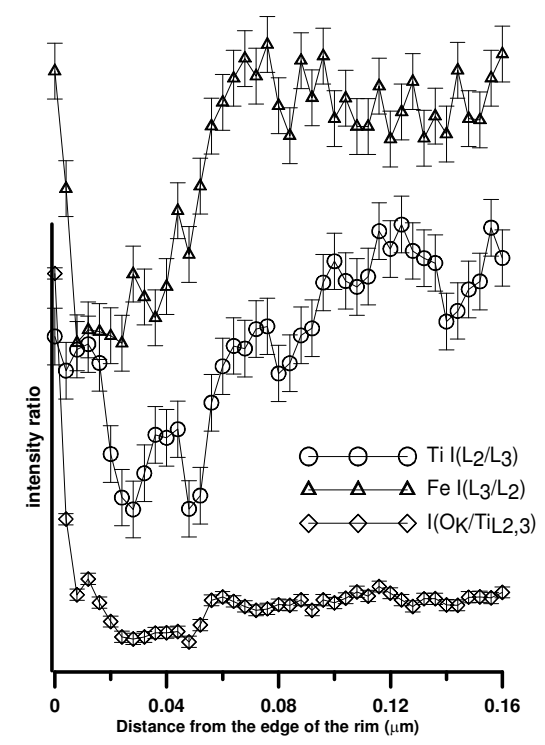

Figure 2. A depth dependent profile showing correlations among $\mathrm{Fe} / \mathrm{Ti}$ valence variation and oxygen distribution in the rim. The rim in the first $20 \mathrm{~nm}$ is formed through vapor deposition, not discussed here. 